\title{
Some characteristics of the outer Oort cloud as inferred from observations of new comets
}

\author{
L. Neslušan and M. Jakubík
}

\author{
Astronomical Institute, Slovak Academy of Sciences, 05960 Tatranská Lomnica, Slovakia \\ e-mail: [ne;mjakubik]@ta3.sk
}

Received 13 September 2004 / Accepted 10 March 2005

\begin{abstract}
We study the outer Oort cloud (OC) by numerical integration of 126600 orbits of hypothetical comets representing this cometary reservoir. We determine the distribution of the semi-major axis $a$ for $4.6 \geqq \log (a) \lesssim 5$, and estimate its population and total mass, as well as some other characteristics. The distribution of the semi-major axis is found initially by assuming a constant $\log (a)$ distribution and then identifying the theoretically predicted $\log (a)$ distribution of new comets with their observed $\log (a)$ distribution. The semi-major axis of outer-OC comets ranges from $\log (a) \approx 4.35$ to typically $\log (a) \approx 5$ on the decadic-logarithm scale. The relative distribution of this element can, however, be roughly determined only for $\log (a) \gtrsim 4.55$ : $\mathrm{d} N \propto a^{-s} \mathrm{~d} a$, where $0.6 \lesssim s \lesssim 0.8$. The perihelion distribution of new comets with $\log (a) \lesssim 4.55$ is not constant in the zone of visibility $(\mathrm{ZV})$ and a bias due to the observational selection cannot be eliminated. The lower limit of the outer OC population is $1 \times 10^{11}$ to $2 \times 10^{11}$. The currently available cometary data do not allow us to give a more exact lower estimate or an average estimate of the population. The OC has also been depleted by the combined Galactic-tide-planetary (GTP) perturbation with a local maximum rate at $\log (a) \approx 4.5$. The ratio of these comets that are lost from the outer OC and of new comets that enter into the $\mathrm{ZV}$ in the same time frame is $\approx 2.5$. The distributions of the angular elements of new comets at their previous perihelion passage are used to evaluate the correctness of our model of the outer OC. Specifically, we calculated the root of mean squares between the corresponding theoretical and observed distributions of $\omega, \Omega$, and $\cos (i)$ resulting in 2-5\% unexplained residua.
\end{abstract}

Key words. comets: general - Oort cloud - planets and satellites: formation

\section{Introduction}

In 1950, Oort revealed a cumulation of original reciprocal semi-major axes at large heliocentric distances that implied the existence of a huge comet reservoir. He suggested that the comets originated in the planetary region and were ejected out to these large distances by planetary perturbations. Due to perturbations by stars passing near the Solar System, the perihelia of some comets in the distant cloud, now named after Oort, have been reduced enough for the comets to become observable. Oort analysed the efficiency of such a perihelion reduction and concluded that the population of the cloud was about $1.9 \times 10^{11}$ cometary nuclei.

Oort's concept of the comet cloud has gradually been developed to include more details, whereby some parameters have been corrected. Concerning the total population, Hills (1981) showed that the abrupt apparent termination of the Oort cloud (OC) at semi-major axis $a \approx 2 \times 10^{4} \mathrm{AU}$ (astronomical units) was due to observational selection. Comets with shorter $a$ are observed only for a relatively brief time after a star has come sufficiently close to the Sun to deflect a new supply of them into orbits that penetrate the planetary system. Hills predicted the existence of an inner reservoir with a population 0 to 100 times that of the outer cloud. It should be emphasized that he estimated the total population both assuming the passing stars as a single outer perturber and assuming the power-law distribution of semi-major axis of cloud orbits with an ad hoc slope index.

Several authors (Heisler \& Tremaine 1986; Morris \& Muller 1986; Bailey 1986; Duncan et al. 1987; Heisler et al. 1987) later proved that the Galactic tidal torque dominates stellar perturbations as a source of new comets in the planetary region. Duncan et al. (1987) simulated the process of the $\mathrm{OC}$ formation and found that the tidal torque contributes roughly $80 \%$ of the flux of new comets. At the same time, they refined the concept of the inner cloud claiming that $20 \%$ of all comets reside in the outer cloud. According to Fernández (1997, Fig. 2), the relative change in perihelion distance of an OC comet due to the tidal force of the Galactic disc is an order or more (depending on the cometary semi-major axis) larger than that caused by stellar perturbations, on average.

Ever since Oort's discovery of the comet cloud, it has been clear that the cloud has to have changed during its existence under the influence of outer perturbers. Oort assumed near passing stars to be the only significant outer perturber; but in 1978, Biermann and later Clube \& Napier (1982), as well as Napier \& Staniucha (1982), noticed another important source of cloud perturbation: giant interstellar molecular 
clouds. These Galactic objects, together with passing stars, had to have strongly eroded the outer region of the comet cloud in particular. The Galactic tide (GT) itself cannot remove comets from the OC, because they are inside the corresponding Hill surface, which has zero velocity relative to the Sun (Heisler \& Tremaine 1986). However, it efficiently reduces cometary perihelia to the planetary perturbation zone and planetary perturbations can then deplete the OC population as well.

Recently, Dones et al. (2004) performed calculations for the "reference model" for OC formation. They integrated the orbits of 3000 hypothetical comets, initially in low-inclination, loweccentricity orbits with semi-major axes of 4-40 AU, for times up to $4 \mathrm{Gyr}$. The simulation included the gravitational influence of the Sun, the four giant planets, GT, and passing stars. More specifically, they performed two sets of runs with dynamically "cold" and "warm" initial conditions. For the "cold" runs, the OC population peaked $840 \mathrm{Myr}$ after the beginning of the simulation. After $4 \mathrm{Gyr}, 2.4 \pm 0.4 \%$ of the original comets reside in the outer OC and $2.7 \pm 0.4 \%$ of the original comets in the inner cloud. For the "warm" runs, the corresponding figures are $1.7 \pm 0.4 \%$ and $3.6 \pm 0.6 \%$, respectively. Both models predict residual populations in the combined Kuiper belt and classical scattered disc, which are comparable to the outer OC population at $4 \mathrm{Gyr}$. In reality, the populations of these reservoirs appears to be $\approx 1 \%$ of the outer OC population. Assuming an outer OC population of $(0.5-1.0) \times 10^{12}$ comets and an average cometary mass of $4 \times 10^{13} \mathrm{~kg}$, their model results imply that the original mass in planetesimals between 4 and 40 AU was $\approx 100-400 M_{\oplus}$ (Earth masses), some 2 to 8 times the mass of solids in a minimum-mass solar nebula.

Previous researchers have derived the population and structure of the OC in two basic ways: (i) through a scenario of its formation, and (ii) modelling its dynamics and gauging the predicted flux of comets in the zone of visibility ( $\mathrm{ZV})$ with the observed flux of dynamically new comets in this region. In this work, we proceed in the second way. More specifically, if we take the perturbers acting on the cloud into account, we can predict the rate of so-called new comets occurring in the $\mathrm{ZV}$. The new comets were not significantly perturbed by the planets at their previous perihelion passage, and their original orbits (i.e. orbits before a comet arrives at the perturbing region of planets at the present perihelion passage) still carry relatively reliable information about the cloud dynamics. Since the new comets in $\mathrm{ZV}$ are observed, their original orbits are known, which allows us to solve an inverse task, from distribution of the reciprocal semi-major axis $1 / a$ of observed new comets, we can gauge the theoretical $1 / a$ distribution of new comets and, consequently, of all comets in an outer-OC model.

On the basis of the model, we further construct the theoretical distribution of perihelion distance of new comets in the $\mathrm{ZV}$, as well as the distributions of their angular elements when they approach the ZV. Further, we estimate the population and corresponding total mass of the outer OC. Subsequently, we discuss an implied total mass of the whole OC and the heavy-chemicalelement component of proto-planetary nebula with respect to a possible upper limit. Moreover, we deal with the depletion rate of the cometary reservoir by the combined Galactic-tide and planetary perturbation and answer a few other questions.
Data on the original orbits of new comets are, unfortunately, not very extensive and, of course, exact. Because of this reason, we attempt to determine the uncertainty of a specific result or, eventually, answer the question of whether a given partial problem can be solved at all with the help of the available data. The uncertainty is roughly appreciated using both older, less numerous and newer, more complete data and then comparing the corresponding results.

\section{Mapping of the evolution of orbits}

In its trajectory through the Galaxy, the OC has been influenced by a number of various factors, themselves often random and unpredictable. In an attempt to make our estimate as reliable as possible, we analyse only the most recent dynamical evolution of OC comets. We assume a steady state comet cloud during the considered period. Such an assumption is reasonable, as the observed pattern of most aphelia of long-period (LP) comets are associated with the GT-linked structure that excludes a strong recent, temporarily effective perturbation of the OC (Fernández 1994).

Furthermore, we only consider the action of the Galactic potential as described by Harrington (1985) and Heisler \& Tremaine (1986), and ignore the other outer (and inner) perturbers on purpose. Reduction of perihelia due to perturbation by both passing stars and interstellar clouds seems to have been negligible in the recent dynamical evolution of the OC (see Sect. 4.4); therefore the picture obtained should not be significantly different from one that considers other perturbers. Moreover, we feel it is useful to profit from both very different concepts of the OC dynamics: first the dynamics under the single influence of GT, in this work, and then the GT together with passing stars and, possibly, interstellar clouds, in future work. After comparing the corresponding results, potential fine effects attributed to individual perturbers should finally be revealed.

\subsection{The initial distribution of orbital elements}

The local Galactic gravitational potential (see Sect. 2.2) has been described in the heliocentric rectangular coordinate system with axes $x$ pointing away from the Galactic centre, $y$ pointing in the direction of Galactic rotation, and $z$ pointing toward the south Galactic pole. Throughout this paper, we use only this coordinate system, referred to as the modified galactic coordinate system.

Assuming the origin of comets in the proto-planetary disc, Duncan et al. (1987) simulated the ejection of comets from this region to large heliocentric distances, where the cometary orbits were perturbed by passing stars and GT. They showed that the spatial distribution of comets, initially inclined toward the ecliptic, was completely randomized by the outer perturbers during $\approx 1$ Gyr. This result leads us to assume a random initial distribution of angular orbital elements. Specifically, we adopt discrete sets of the initial argument of perihelion $\omega_{0}$ and initial longitude of galactic ascending node $\Omega_{0}$, both varying from $15^{\circ}$ to $345^{\circ}$ with step $30^{\circ}$. Similarly, we adopt the discrete 
set of cosines of the initial inclination $\cos \left(i_{0}\right)$ varying from -0.9 to +0.9 with step 0.2 .

In the averaged Hamiltonian corresponding to the function expressing the local Galactic potential, the semi-major axis of an orbit of Oort-cloud comet $a$ is constant (Heisler \& Tremaine 1986). There is no significant difference between the values of $a$ at large heliocentric distances and at a perihelion in the planetary region. The real distribution of this element in the cloud can thus be traced from its observed distribution in the ZV. In our set of orbits of hypothetical comets, we assume the discrete values of their initial semi-major axis in a decadic logarithmic scale $\log (a)$, ranging from 4.2 to 5.0 with step 0.1 , where $a$ is given in AU. The values from 4.4 to 5.0 cover the interval of $\log (a)$ for observed new comets. Values 4.2 and 4.3 are considered to study the inner border of the outer OC in more detail along with the rate of depletion of the OC in this region. Initially, we consider a constant (flat) distribution of $\log (a)$; the same number of comets is assumed for each value of $\log (a)$ considered. When the number of new comets with individual $\log (a)$ is predicted and compared with the corresponding actual number of new comets in the catalogue, both the numbers differ by a factor. The latter is then utilized as a multiplication coefficient to equalize the theoretical number with its observed counterpart. The coefficients for every $\log (a)$ characterize the real $\log (a)$ distribution of comets in the outer OC.

To complete our assumption of the initial cloud-comet elements, it is still necessary to assume the distribution of a fifth element: either perihelion distance or eccentricity. Jeans (1919) studied the origin of stellar binary systems. Assuming that the orbits of older binaries have reached something at least approximating a final state of equipartition of energy, he derived that all values of the eccentricity quadrate $e^{2}$ were equally probable. Hills (1981) applied the same assumption to OC comets in order to estimate their total population. We adopt this assumption, too, to obtain the initial distribution of eccentricity $e_{0}$; therefore, we consider a set of discrete initial values of $e_{0}^{2}$ in a range from 0.05 to 0.95 with step 0.1 . The values represent the centres of intervals from 0 to 0.1 , from 0.1 to 0.2 , etc. It should perhaps be stressed that there is a major difference between Hills's and our assumption of the same distribution law for eccentricity. While Hills derived the amount of bodies filling a so called "loss cone", we use it to create the initial distribution of orbits, which are subjects to further dynamical evolution.

The above distribution law for eccentricity was also assumed by Matese et al. (1995), who supposed random distribution of comet angular momenta and perihelia directions. It should be noted that assumption of random distribution of angular orbital elements and mutual independence of individual elements was criticized by Dybczyński \& Pretka (1996). They pointed out the well-known fact that changes in the orbital elements of cloud comets, being perturbed by the GT, are synchronized, therefore these elements might not be distributed randomly. Following this criticism, we choose a random distribution of angular orbital elements and the $e^{2}$ distribution law only at the beginning of dynamical evolution, which we follow through a numerical integration of the chosen grid of orbits. Performing the integration during a sufficiently long period of
5 Gyr, we attempt to find the distribution of orbital elements in the appropriate five-dimensional phase space and, at the same time, to map their above-mentioned coupling.

We record two kinds of output from the integration: (i) the osculation orbital elements at each perihelion passage of every hypothetical comet and (ii) all osculation orbital elements recorded with a step of $1 \mathrm{Myr}$.

\subsection{The numerical integration}

The numerical integration of each orbit from the initial set, as described in Sect. 2.1, is performed using the well-known description of Galactic tidal force $\boldsymbol{F}$, suggested at first by Antonov \& Latyshev (1972) and later refined by Harrington (1985) and by Heisler \& Tremaine (1986). According to Heisler \& Tremaine $\boldsymbol{F}=\left\{K_{x} x, K_{y} y, K_{z} z\right\}$, where constants $K_{x}$, $K_{y}$, and $K_{z}$ are equal to $K_{x}=(A-B)(3 A+B), K_{y}=-(A-B)^{2}$, and $K_{z}=-\left[4 \pi G \rho-2\left(B^{2}-A^{2}\right)\right] . A$ and $B$ are the Oort constants, $\rho$ is the mean mass density in the solar neighbourhood, $G$ is the gravitational constant, and $x, y, z$ are the rectangular heliocentric coordinates of a given test particle in the modified galactic coordinates. We perform the direct integration of the equations of motion. The appropriate equations of motion can analytically be integrated once, giving the integration constant $h$, which represents the conserved energy of the system. We then utilize this constant to check the stability of the numerical integration.

As for the mean mass density $\rho$, no precise value is known. Crézé et al. (1998) and Holmberg \& Flynn (2000) published values 0.08 and $0.10 M_{\odot} \mathrm{pc}^{-3}$, respectively, derived from the Hipparcos data. They concluded that there is no compelling evidence for significant amounts of dark matter in the disc. However, García-Sánchez et al. (2001) revealed an incompleteness in the Hipparcos data, which implies a larger actual value of $\rho$. Several authors in the past used a value of $0.185 M_{\odot} \mathrm{pc}^{-3}$ found by Bahcall (1984). Holmberg \& Flynn presented a summary of the estimates of this quantity spanning from 0.08 to $0.26 M_{\odot} \mathrm{pc}^{-3}$ with a straight average of $0.15 M_{\odot} \mathrm{pc}^{-3}$. The latter was recently used by Fernández (1997) and Wiegert \& Tremaine (1999), and is again used for this paper. We also take the same values of the Oort constants as Wiegert \& Tremaine, $A=14.4 \mathrm{~km} \mathrm{~s}^{-1} \mathrm{kpc}^{-1}, B=-12.0 \mathrm{~km} \mathrm{~s}^{-1} \mathrm{kpc}^{-1}($ Kerr \& Lynden-Bell 1986).

Due to the uncertainty of $\rho$, the inner border of the dynamically active outer $\mathrm{OC}$ at $\log (a) \approx 4.35$ is not reliably determined. We must realize that a lower value of the density would result in a more distant border, while a higher value results in a border nearer to the Sun. Further research is still necessary to determine the actual distance of this border more precisely.

Heisler \& Tremaine (1986) discovered the periodic variations of orbital elements with a period up to several gigayears at the inner border of the outer cloud; for the transparent graphical behaviours of elements also see Pretka \& Dybczyński (1994). To map the five-dimensional phase space of orbital elements, characterizing the position and shape of the orbit properly, we perform the numerical integration of the orbits of each hypothetical comet over 5 Gyr. We would like to emphasize 
that we choose such a long integration period to map the recently evolved phase space, not to follow the orbital evolution during the age of the Solar System, which is a much more complicated task as the system has changed its position in the Galaxy.

Since solar gravity clearly dominates over the considered tidal force, a given hypothetical comet moves upon a trajectory very similar to a Keplerian orbit. In this circumstance, it is both possible and time-saving for computation to choose a variable integration step $\Delta t$, proportional to the quadrate $r^{2}$ of the heliocentric distance of the comet; or in more detail, $\Delta t=\Delta t_{0} r^{2}$, where $\Delta t_{0}$ is a constant arbitrarily chosen at the beginning of integration. We have chosen a compromise between the precision and computation time with such $\Delta t_{0}$ that the relative change of constant $h$ never exceeds 0.001 (it is typically one order lower) over the entire integration period, $t_{\mathrm{IP}}=5 \mathrm{Gyr}$.

\section{On the observational data}

\subsection{New comets in the visibility zone}

Due to GT perturbation, some comets from the outer Oort cloud enter the ZV. The extent of the cometary population can only be deduced from information gained from observations of these bodies. So, the basic task of our study is to determine what fraction of comets crosses the central part of the Solar System per unit of time.

In the past, comets with $1 / a<10^{-4} \mathrm{AU}^{-1}$ were regarded as dynamically new, unperturbed by the planets before their observed (current) entry to the ZV. Dybczyński (2001), however, showed that this criterion is insufficient because 41 of 85 comets it classified as new were likely to be significantly perturbed by the planets at their previous perihelion passage if the original $a$ value was accurate. He regarded as new only those comets that had their perihelion distances $q$ beyond $15 \mathrm{AU}$ at the previous perihelion passage. This distance was found to be critical by Duncan et al. (1987), who concluded that comets with pericentres less than about $15 \mathrm{AU}$ were unlikely to survive for the age of the Solar System. The planetary perturbations are so large that the GT does not have time to torque them out of the planetary region before they are ejected.

To eliminate observational selection effects on comet discoveries as much as possible, we do not consider new comets with current perihelia beyond a certain border of heliocentric distance $q_{\text {out }}$, where the available sample of observed bodies is too low because of observational selection (i.e., not enough comets are included in the data). Thus a new comet has to satisfy the following criterion: the $q$ of its original orbit at the current perihelion passage must be $q \leq q_{\text {out }}$, and $q$ of its orbit at the previous perihelion passage has to be situated beyond $15 \mathrm{AU}$.

\subsection{The available original orbits}

The most numerous sample of the original orbits has recently been published in the Catalogue of Cometary Orbits, 15th edition (Marsden \& Williams 2003). The orbits are divided into two classes by quality. Unfortunately, no quantitative error is given for the determination of original $1 / a$. Values of the original $1 / a$ with the determination errors can be found in several papers by Marsden and coauthors, but only for a smaller sample from the original orbits.

Since no available data sample is statistically numerous enough, we try to extract each accessible piece of information about the outer OC comparing results based on the following two samples and their subsets:

(i) We use 249 original orbits published in six papers by Marsden and coauthors (Marsden \& Sekanina 1973; Van Biesbroeck et al. 1974; Marsden et al. 1978; Everhart \& Marsden 1983, 1987; Marsden 1990). The known standard deviation $\sigma_{1 / a}$, of $1 / a$ for each of these orbits, allows us to construct an error Gaussian giving a distribution probability of $1 / a$ around the published value. The total area under the Gaussian is set to unity. A given interval of $\log (a)$ contains a certain part of the Gaussian area, which is assumed to be the weight of the contribution by a given comet to this interval. The sample with the weighted contributions is referred to as the "Gaussian-area-contribution (GAC) sample".

(ii) Moreover, we use the reciprocal semi-major axes, perihelion distances, and inclinations to the ecliptic of 386 original orbits of LP comets published in the Catalogue of Cometary Orbits, 15th edition (Marsden \& Williams 2003). Three orbits are excluded because of their similarity to another three and their probable splitting origin (for details see paper by Neslušan \& Jakubík 2004, Sect. 2.1, hereinafter referred to as Paper I). Because the determination errors have not been published, we are forced to consider the contribution of each comet with a unit weight. The sample is referred to as the "Catalogue-of-CometaryOrbits (CCO) sample".

Apart from considering all (ALL) comets in a given sample, we check to see if the features we found can also be observed in the subset of comets in orbits of higher, class 1 (CL1) quality. The sets of the original elements of the orbits in both samples are completed with the original angular elements calculated by Dybczyński (private communication) utilizing the method described in his work published in 2001.

To select the new comets, the orbits in each set are integrated back to their previous perihelion passage, or up to the heliocentric distance $10^{6} \mathrm{AU}$ in the case of hyperbolicity, taking the perturbation of the GT into account. In the GAC sample, we integrate back not only the orbits with the published original $1 / a$ but also those orbits with modified $(1 / a)$ s from the interval ranging from $(1 / a)_{5.05}$ to $(1 / a)_{4.25}$, step $1 \times 10^{-6} \mathrm{AU}^{-1}$, unless the weight corresponding to given $1 / a$ is less than 0.001 . The value of $(1 / a)_{4.25}$ corresponds to the lower border of the first $\log (a)$ interval $(\log (a)=4.25)$ considered while value of $(1 / a)_{5.05}$ corresponds to the upper border of the last considered $\log (a)$ interval $(\log (a)=5.05)$. A given modified orbit contributes to the constructed distributions with weight corresponding to the part of Gaussian area over the $1 / a$ interval of width equal to the $1 / a$-modification step with the centre at the modified $1 / a$. In this way, the comets in the hyperbolic original orbits also contribute to the constructed distributions, when the 
GAC sample is considered. When the CCO sample is considered, the hyperbolic comets must simply be ignored because there is no "previous" perihelion passage and the criterion for the selection of new comets cannot be satisfied.

We find that 53 comets in the CCO sample satisfy the criterion for the orbits of new comets. The sum of weights of those orbits which satisfy this criterion in the GAC sample is 24.070 .

\subsection{The influence of observational selection}

We can expect that the set of observed new comets is strongly affected by observational selection effects on comet discoveries and original orbit determinations. In a first attempt to eliminate selection effects as much as possible, we do not consider new comets with current perihelia beyond the already mentioned border of heliocentric distance $q_{\text {out }}$. Constructing the distribution of current $q$ for new comets selected from the GAC (CCO) sample, it is possible to find that $q$ spans from 0 to about $7 \mathrm{AU}$ (12 AU). However, there are only a few comets with $q>5 \mathrm{AU}$ ( $q>6.5 \mathrm{AU})$; therefore, we set the outer boundary of $\mathrm{ZV}$ to $q_{\text {out }}=5 \mathrm{AU}$ for the GAC sample and to $q_{\text {out }}=6.5 \mathrm{AU}$ for the CCO sample.

Let us denote the true frequency of new comets crossing the $\mathrm{ZV}$ with the $j$ th value of $\log (a)$ by $\Phi_{O j}$ and the apparent frequency of such comets derived from the available data by $\Phi_{D j}$. If $\eta_{j}$ is the rate of completeness of comets with the original semi-major axis from the $j$ th interval in the data, then

$\Phi_{D j}=\eta_{j} \Phi_{O j}$.

The GAC sample used contains comets with perihelion passage from 1822 (one comet in 1811) through 1989, and the CCO sample contains comets passing the perihelion from 1822 (one comet in 1811) through 2002. They yield observational periods $t_{\mathrm{OP}}=168$ and 181 years, respectively. One easily deduces that the number of observed new comets in the catalogue with the $j$ th value of $\log (a), n_{\mathrm{obs} ; j}$, is equal to

$n_{\mathrm{obs} ; j}=\Phi_{D j} t_{\mathrm{OP}}$.

To calculate the true frequency $\Phi_{O j}$ using $n_{\text {obs } ; j}$, we need to determine the rates of completeness $\eta_{j}$ for every $j$ corresponding to the outer OC.

There are, however, some difficulties in the determination of the rates. All new comets have a very large semimajor axis; therefore, every new comet moves in $\mathrm{ZV}$ upon a trajectory practically identical to a part of the corresponding parabolic orbit with the same $q$ and angular orbital elements. Consequently, the time behaviour of two comets in orbits with different $\log (a)$, but the same $q$ and angular elements, is practically identical, and observational selection does not statistically prefer the discovery of a comet with any specific $\log (a)$ unless there is no dependence by $q$ or angular elements on $a$. We know no reason for a dependence of the angular elements on the semi-major axis of LP comets. However, it must be expected at the perihelion distance.

The invariance/dependence of $q$ on $a$, derived from our model of OC described theoretically in Sect. 2, can be seen in Fig. 1. It is well known that the GT typically changes the perihelia of outer-OC comets by thousands of astronomical units.
In the relatively narrow interval of possible $q$ in the $\mathrm{ZV}$, random circumstances predominantly cause the previous perihelion of a new comet to be, for example, $q_{\text {prev. }}=19 \mathrm{AU}$ and its current perihelion, $q_{\text {curr. }}=4 \mathrm{AU}$, or to be $q_{\text {prev. }}=16 \mathrm{AU}$; and it is reduced about the same $\Delta s=15 \mathrm{AU}$ to $q_{\text {curr. }}=1 \mathrm{AU}$. Thus, the occurrence of $q$ of a given comet, with $a$ larger than a certain limiting $a_{r . q \text {. }}$, is random in the $\mathrm{ZV}$; consequently, the distribution of $q$ of new comets in a given interval of $\log (a)$ beyond $\log \left(a_{\text {r.q. }}\right)$ must be random. Actually, we can see practically random behaviour of the perihelion-distance distribution in plots $\mathrm{c}-\mathrm{h}$ in Fig. 1 corresponding to the $\log (a)_{j}$ intervals for $j=2,3,4, \ldots, 7$ (for numbering of the intervals see Table 1). Fitting the linear function $N_{\mathrm{r}}=C q+D$, (where $N_{\mathrm{r}}$ is the relative number of comets and $C$ and $D$ are fitted parameters), one can find that the standard deviation $\sigma_{C}$ of the determination of parameter $C$ is larger than the absolute value of $C$ in each of these behaviours. Therefore, a constant or random behaviour is within $1 \sigma_{C}$ of the fitted function.

In Paper I, we found the behaviour of the average magnitude of perihelion-distance change per one orbital revolution $\langle\Delta s\rangle$ as a function of $a$. As stated there, our result was consistent with that of Fernández (2002). According to the result, only the perihelion of a comet with $a \geq 3.34 \times 10^{4} \mathrm{AU}$ $(\log (a) \geq 4.52)$ can be reduced by the GT from a region beyond the Jupiter-Saturn perturbative region to the $\mathrm{ZV}$ within one orbital period. Thus, $\log \left(a_{\text {r.q. }}\right) \approx 4.52$, and a certain dependence of $q$ on $a$ should also be observed in Fig. 1c for the interval of $\log (a)=4.5$ on the basis of this result. Maybe the dependence is obscured when averaging the magnitude of GT perturbance over angular elements. A consequent dispersion of the magnitude can weaken this dependence and produce a practically non-increasing distribution. Despite the invariance of $q$ on $a$ in Fig. 1c, we take the older value $\log \left(a_{\text {r.q. }}\right)$ into account and assume random distribution of $q$ only in $\log (a)_{j}$ intervals for $j=3,4,5,6$, and 7 . The nature of the $q$ distribution of new comets with $\log (a)$ in $j=2$ interval remains questionable. Since the distribution of $q$ in the ZV of comets in $\log (a)_{j}$ intervals is random for $j=3$ to 7 , the selection effects affect the numbers of new comets observed in the $\mathrm{ZV}$ in the same way in these $\log (a)_{j}$ intervals. As for the completeness rate of comets, we can put $\eta_{3} \approx \eta_{4} \approx \ldots \approx \eta_{7} \equiv \eta_{3-7}$ as a consequence.

Below $a_{\text {r.q. }}$, an occurrence in the $\mathrm{ZV}$ of new comets with given $a$ depends on their perihelion distance (Matese \& Lissauer 2002; Whitmire \& Matese 2003). The perihelia of the new comets cannot be situated below an inner boundary. This boundary is expected to be further and further from the Sun for comets with shorter and shorter $a$. For semi-major axis $a$ shorter than a certain critical value $a_{\text {l.q. }}$, the GT is no longer efficient for reducing the perihelion from a distance larger than or equal to $15 \mathrm{AU}$ to the $\mathrm{ZV}$, i.e. to a distance shorter than or equal to $6.5 \mathrm{AU}$ (or $5 \mathrm{AU}$ for the GAC sample). Actually, only a few new comets with $q$ at the outer boundary of $\mathrm{ZV}$ are seen in Fig. 1a. The distribution of perihelion distances of new comets, with $a \in\left(a_{l . q .}, a_{r . q}\right)$, is obviously dependent on $a$ as we can expect fewer and fewer new comets with shorter and shorter perihelion distance, in this case. The decrease in the number of new comets with decreasing $q$ is seen clearly in Fig. $1 \mathrm{~b}$. When the linear function $N_{\mathrm{r}}=C q+D$ is fitted to the behaviour, one 

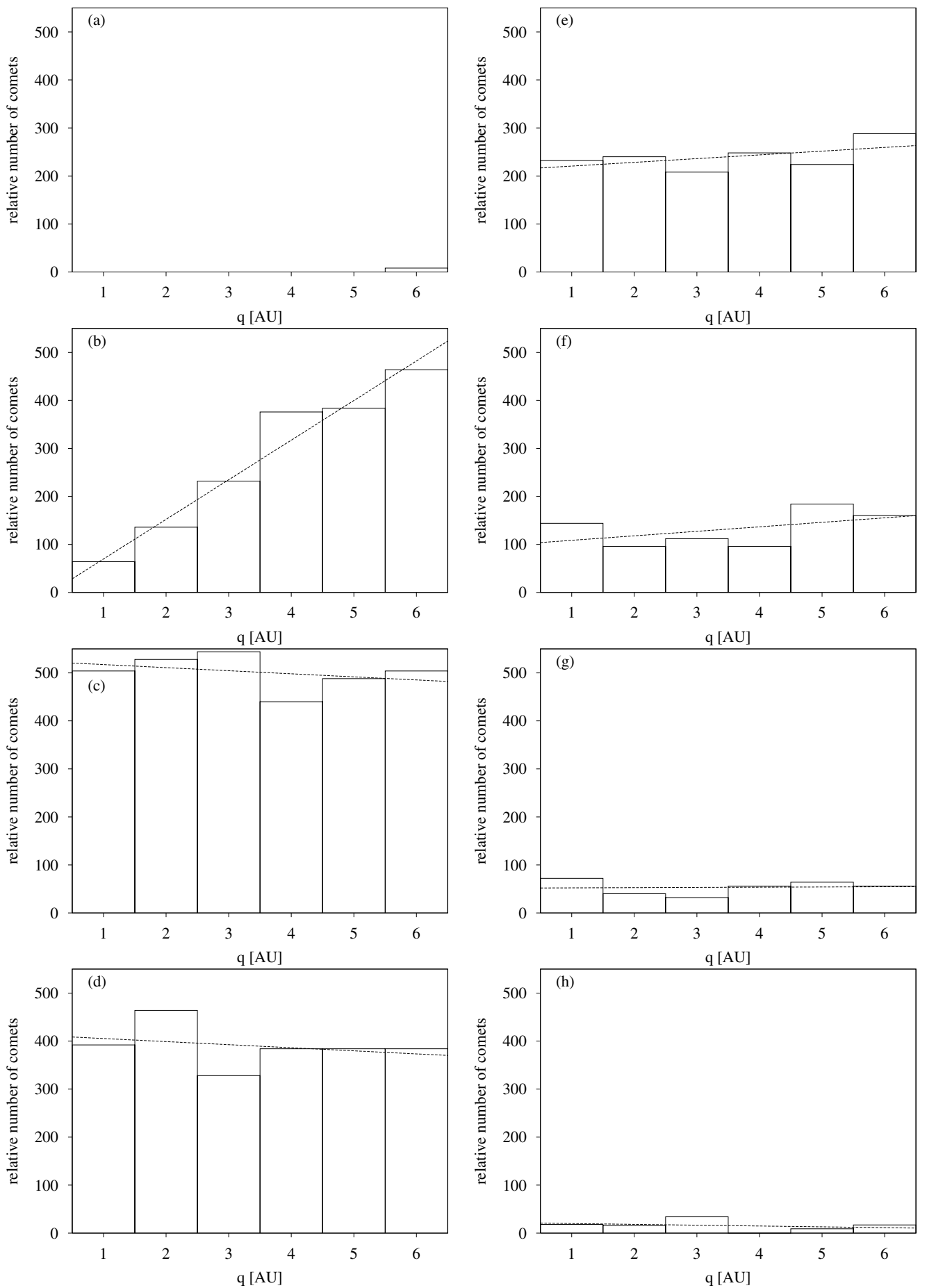

Fig. 1. The theoretical distributions of perihelion distance $q$ of new comets in the ZV in the intervals of $\log (a)$ corresponding to the outer part of the Oort cloud. The distributions, derived from the model of the cloud described in Sect. 2, are given for the intervals centred at log $(a)=4.3$, $4.4,4.5, \ldots, 5.0$ in plots $\mathbf{a}), \mathbf{b}), \mathbf{c}), \ldots, \mathbf{h})$, respectively. A linear function is fitted to the behaviours in plots $\mathbf{b})-\mathbf{h})$.

can find that $C=83 \pm 12$. The parameter $C$ corresponding to a constant behaviour is not situated even within $3 \sigma_{C}$ in this case.

\subsection{Mean reciprocal semi-major axis vs. perihelion distance}

The dependence of mean reciprocal semi-major axis on the perihelion distance in the $\mathrm{ZV}$ was analysed by Matese \& Lissauer (2002). An actualized plot can be found in a paper by Whitmire \& Matese (2003, Fig. 2). The same dependence, but constructed for the parameters adopted in this work, can check the agreement of our theoretical model of OC with observations.

Using the CCO-ALL sample, the comparison between the predicted and observed mean 1/a vs. $q$ is illustrated in Fig. 2. The predicted behaviour (dashed line) is well within $1 \sigma$ (standard error) of the catalogue mean $1 / a$ values calculated for $q$ 


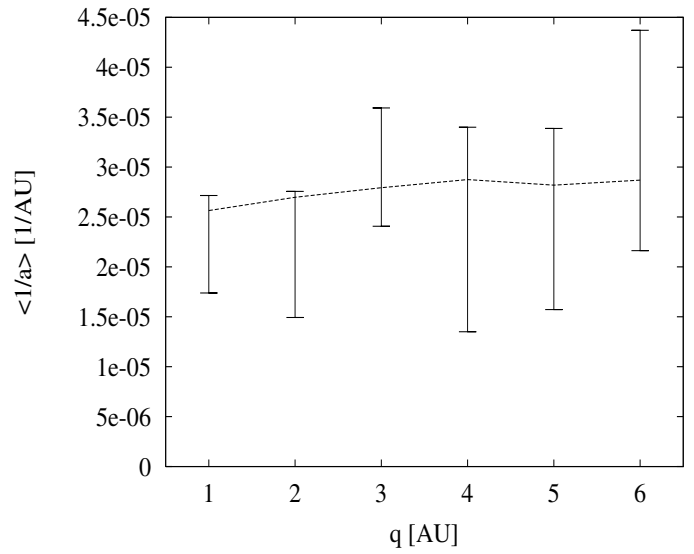

Fig. 2. The dependence of mean reciprocal semi-major axis $\langle 1 / a\rangle$ on the perihelion distance $q$ in the $\mathrm{ZV}$. This dependence is calculated from the $(1 / a)$ s of new comets in the entire (ALL) CCO sample for $q$ intervals of width equal to $1 \mathrm{AU}$ (vertical bars) and also predicted on the basis of our model of the outer OC (dashed line). The length of each bar represents the $2 \sigma$ (a deviation $\pm \sigma$ ) uncertainty of the $\langle 1 / a\rangle$ determination.

intervals of $1 \mathrm{AU}$ width (vertical bars representing the possible $\pm 1 \sigma$ range). The same fact can be stated for the other data subsets under consideration.

The standard error here is generally larger than the one in the paper by Whitmire \& Matese (2003). It is obviously a consequence of using a different criterion in the choice of new comets from the catalogue. Whitmire \& Matese used the older, $1 / a<10^{-4} \mathrm{AU}^{-1}$ criterion giving a larger number of new comets, which leads to a formally smaller standard error.

\section{The distribution of semi-major axis in the outer Oort cloud}

\subsection{A construction of the relative distribution of $\log (a)$}

In the hypothetical set of numerically integrated orbits with given $\log (a)$, we consider orbits with every combination of 12 values of $\omega, 12$ values of $\Omega, 10$ values of $\cos (i)$, and 10 values of $e^{2}$ (Sect. 2.1), i.e. a set of $N_{\mathrm{all} ; j}=14400$ orbits for every $j$.

During the integration period $t_{\mathrm{IP}}$, a number $N_{\mathrm{nf} ; j}$ (Table 2) of all $N_{\text {all } ; j}$ hypothetical comets come to the $\mathrm{ZV}$, where they can be identified with the actually observed new comets. The number $N_{\mathrm{nf} ; j}$ per time $t_{\mathrm{IP}}$ can be regarded as the rate of new comets in the analyzed phase space. Of course, the rate differs from the actually observed number of new comets as the actual distribution of $\log (a)$ in the OC is not obviously identical to the constant $N_{\text {all } ; j}$ distribution. The real $j$ th group of comets does not consist of $N_{\text {all; } j}$ bodies, but it truly consists of $N_{\text {true; } j}$ bodies, whereby

$N_{\text {true } ; j}=w_{j} N_{\text {all } ; j}$.

The symbol $w_{j}$ stands for the multiplication coefficient of the $j$ th group to convert the constant $\log (a)$ distribution to the actual $\log (a)$ distribution. Coefficients $w_{j}$ for every considered
Table 1. The observed decadic logarithm distribution for the semimajor axis of new comets, $n_{\mathrm{obs} ; j}$. Specifically, $n_{\mathrm{obs} ; j}$ is the number of observed (i.e. contained in the catalogue) new comets in the $j$ th interval of $\log (a)$. The distribution is given for both subsets, ALL and CL1, of the GAC and CCO samples of long-period comets (see Sect. 3.2 for a detailed description).

\begin{tabular}{cccccc}
\hline \hline & & \multicolumn{2}{c}{ GAC } & \multicolumn{2}{c}{ CCO } \\
$j$ & $\log (a)_{j}$ & ALL & CL1 & ALL & CL1 \\
\hline-1 & 4.2 & 0 & 0 & 0 & 0 \\
0 & 4.3 & 0.024 & 0.024 & 0 & 0 \\
1 & 4.4 & 1.464 & 1.363 & 8 & 8 \\
2 & 4.5 & 5.666 & 5.129 & 10 & 9 \\
3 & 4.6 & 7.383 & 6.878 & 15 & 14 \\
4 & 4.7 & 5.624 & 5.190 & 10 & 9 \\
5 & 4.8 & 2.535 & 2.327 & 5 & 5 \\
6 & 4.9 & 1.149 & 1.077 & 3 & 2 \\
7 & 5.0 & 0.249 & 0.230 & 1 & 1 \\
\hline
\end{tabular}

Table 2. The theoretical distribution of decadic logarithm of semimajor axis of new comets, $N_{\mathrm{nf} ; j}$. Specifically, $N_{\mathrm{nf} ; j}$ is the predicted (theoretical) number of new comets in the zone of visibility with $\log (a)$ in the $j$ th interval, when the number of all considered hypothetical comets in the $j$ th interval is $N_{\text {all } ; j}$. The distribution is given for both considered samples, GAC and CCO, of long-period comets (see Sect. 3.2 for a detailed description). (It is the same for the entire, ALL, sample and subset CL1.)

\begin{tabular}{cccc}
\hline \hline$j$ & $\log (a)_{j}$ & $\mathrm{GAC}$ & $\mathrm{CCO}$ \\
\hline-1 & 4.2 & 0 & 0 \\
0 & 4.3 & 0 & 8 \\
1 & 4.4 & 1072 & 1696 \\
2 & 4.5 & 2544 & 3376 \\
3 & 4.6 & 1928 & 2496 \\
4 & 4.7 & 1184 & 1616 \\
5 & 4.8 & 576 & 840 \\
6 & 4.9 & 232 & 328 \\
7 & 5.0 & 87 & 104 \\
\hline
\end{tabular}

$j$ can be calculated to identify the theoretical frequencies of the occurrence of new comets in ZV with their actually observed counterparts. The $j$ th theoretical frequency can be given as $w_{j} N_{\mathrm{nf} ; j} / t_{\mathrm{IP}}$. Therefore,

$\frac{w_{j} N_{\mathrm{nf} ; j}}{t_{\mathrm{IP}}}=\Phi_{O j}$.

From Eqs. (1)-(4), we obtain the actual number of comets with given $\log (a)$ in the outer OC

$N_{\text {true } ; j}=\frac{1}{\eta_{j}} \frac{t_{\mathrm{IP}}}{t_{\mathrm{OP}}} \frac{N_{\mathrm{all} ; j}}{N_{\mathrm{nf} ; j}} n_{\mathrm{obs} ; j}$.

The numbers $N_{\mathrm{nf} ; j}$ can be counted in the resulting data of numerical integration described in Sect. 2; i.e. in the derived model of the OC, both $n_{\mathrm{obs} ; j}$ and $N_{\mathrm{nf} ; j}$ are given in Tables 1 and 2 for the two considered samples of original orbits, GAC and CCO, as a whole (set ALL) as well as their subset CL1. In the sample CCO, one orbit with $\log (a)>5.05$ is ignored.

Assuming a constant rate of completeness in the catalogue for all non-empty $\log (a)$ intervals, i.e. $\eta_{1}=\eta_{2}=\eta_{3}=\ldots=\eta_{7}$, we can use Eq. (5) to calculate the relative $N_{\text {true; } j}$ and construct 

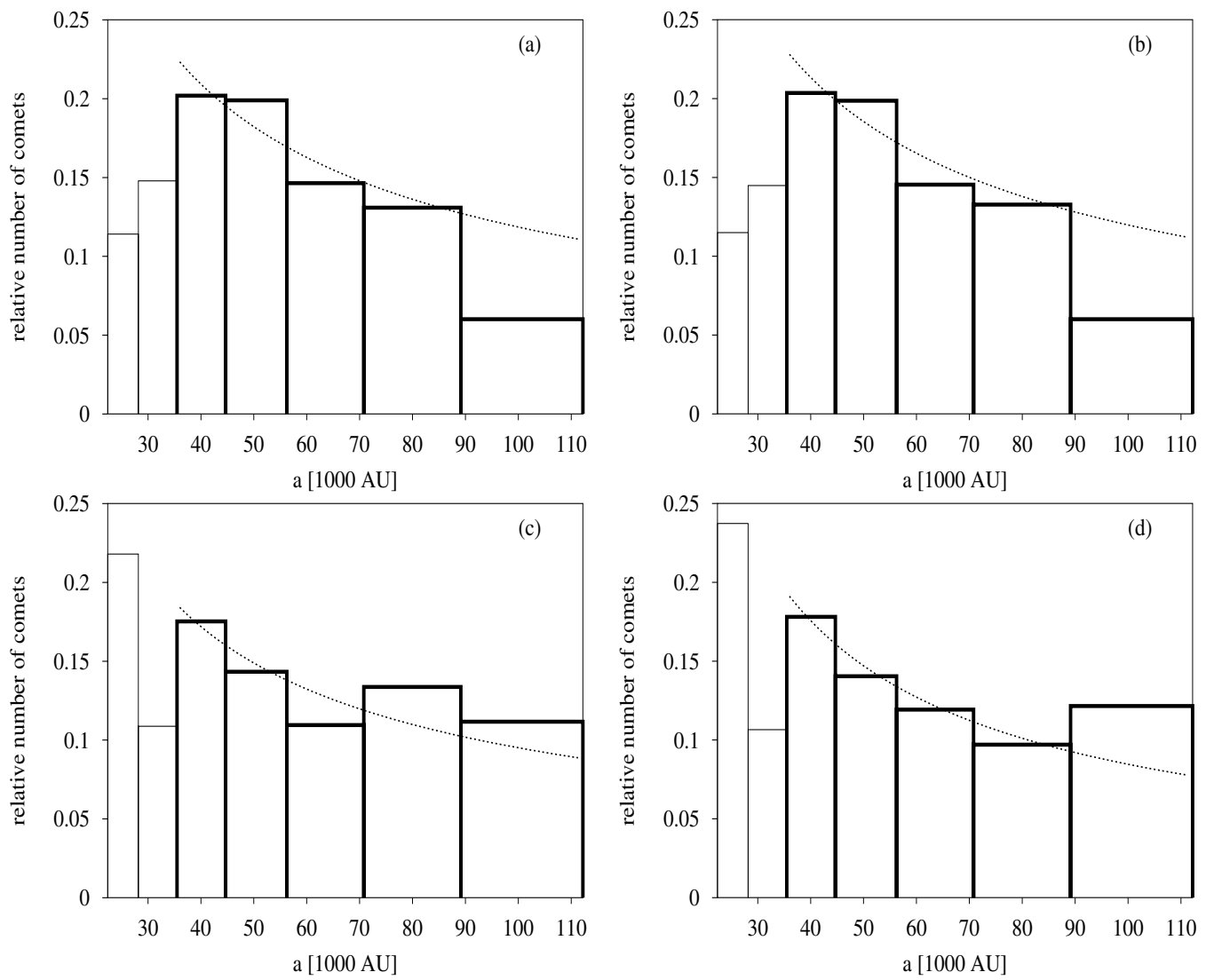

Fig. 3. The true relative distribution of the semi-major axis $a$ of orbits in the outer Oort cloud up to $\log (a)=5.05(a$ is given in AU) constructed using the GAC (a), b)) and CCO (c), d)) sample (see Sect. 3.2) of the original orbits of long-period comets. The left-column plots, a) and c), illustrate the distribution for the entire (ALL) sample and the right-column plots, b) and d), illustrate it for the subset of quality-class-1 orbits (CL1). The distribution is a linearization of the corresponding $\log (a)$ distribution. The two values (thin-line bars) to the left in each plot for $\log (a)=4.4$ and 4.5 are lower estimates of the actual relative numbers. The other five values (thick-line bars) represent a normal estimate. The dashed curve is a fit of the power law to the five estimated weighted relative numbers.

the relative true distributions of the semi-major axis of comets in the outer, dynamically active OC using each of the considered samples and their subsets of observational data. The distributions are shown in Fig. 3 for the considered data samples.

As discussed in Sect. 3.3, the rate of catalogue completeness can be regarded as constant for $\log (a)_{j}$ intervals with $j \geq 3$ (thick-line bars in Fig. 3). To provide the most general behaviour feature of the distribution of $a$ (linearized $\log (a)$ ) in the range corresponding to $j \geq 3$, we fit the power-law function $N_{\mathrm{r}}=E(a / 1000)^{-\tau}$ ( $E$ is a parameter and $\tau$ the index of slope) to all constructed distributions. In the fitting, a weight proportional to the number of observed new comets in the given $\log (a)$ interval, used in gauging the $\log (a)$ distribution, is assigned to the corresponding value of $N_{\mathrm{r}}$. It suppresses the relatively larger statistical fluctuations in the intervals containing only a few comets.

The numerical values of $E$ and $\tau$ are given in Table 3. The determination errors of these parameters can be formally calculated, of course, but they are much smaller than the differences between the values of a given parameter determined using the different basic data samples/subsets. The formal errors thus have no actual information value so we do not present them. The differences between the corresponding values are obviously caused by the different selection effects on the
Table 3. The coefficient and slope index of the power-law function $N_{\mathrm{r}}=E(a / 1000)^{-\tau}$, fitted to the distribution of semi-major axis $a$ of OC comets in the interval $\log (a) \in(4.55,5.05) . N_{\mathrm{r}}$ is the relative number of comets.

\begin{tabular}{ccccc}
\hline \hline & \multicolumn{2}{c}{ GAC } & \multicolumn{2}{c}{ CCO } \\
& $E$ & $\tau$ & $E$ & $\tau$ \\
\hline ALL & 2.06 & 0.62 & 1.87 & 0.65 \\
CL1 & 2.18 & 0.63 & 3.34 & 0.80 \\
\hline
\end{tabular}

individual samples and subsets of considered data. They can serve to guess the uncertainty of the determination of the parameters.

The index of slope $\tau$ varies from $\approx 0.62$ to $\approx 0.65$ for all considered subsets apart from the CCO-CL1, where $\tau \approx 0.80$. Even this higher value, however, indicates that the $\mathrm{OC}$ is less centrally concentrated than assumed by Hills (1981). He considered the models with $\tau=2$ and $\tau=2.5$.

The relative numbers of comets in $\log (a)_{j}$ intervals for $j=1$ and 2 (thin-line bars in Fig. 3) can be regarded as minimum numbers. The perihelia of new comets with $\log (a)$ in these intervals are over more concentrated towards the outer boundary of $\mathrm{ZV}$, where fewer and fewer comets are discovered due to their fainter brightness. In other words, a relative 
lower number of new comets with $\log (a)_{j}$ for $j=1$ and 2 have been discovered than new comets with $\log (a)_{j}$ of larger $j$. Consequently, the actual rates of completeness $\eta_{1}$ and $\eta_{2}$ are not equal to $\eta_{3-7}$, but $\eta_{1}<\eta_{2}<\eta_{3-7}$.

The nature of the local minimum of relative number for $j=$ 2 seen in Fig. 3, plots $\mathrm{c}$ and d, remains an open question. If the decrease in the number from $j=3$ to $j=2$ was caused simply by a lack of new comets with a larger $q$ in the $\mathrm{ZV}$, then it should continue from $j=2$ to $j=1$, as seen in the less numerous GAC database in Fig. 3, plots a and b. But the data completed with the new comets that have been discovered in the most recent period between 1989 and 2002 and that have a relatively large $q$ yield the existence of a high concentration of comets at the innermost border of that part of OC from which new comets are currently observable (the highest relative number, for $j=1$, in Fig. 3, plots c and d). A possible reason for a reality of the minimum is discussed further in Sect. 4.4. In conclusion, we do not have enough evidence at present, however, to exclude the possibility that the relative numbers for $j=1$ and 2 are strongly underestimated and that there is a continuous increase from higher to lower $j$. A relatively large increase from $j=2$ to $j=1$, seen in Fig. 3 plots $\mathrm{c}$ and d, would then indicate an extreme real increase in the number of comets in this region of OC.

\subsection{Distribution of angular elements of new comets}

In the previous section, we outlined the distribution uncertainty of the semi-major axis in the outer OC constructed using the data available at present. After replacing the initially assumed constant $\log (a)$ distribution (Sect. 2.1) with the found $\log (a)$ distribution, we obtain a model describing the outer OC. The quality of the approximation of reality by this model can be appreciated when constructing the theoretical distributions of the angular elements of new comets coming to the $\mathrm{ZV}$, and when comparing them with their observed counterparts. If all the assumed initial distributions of cometary orbits determining the model are correct, the corresponding theoretical and observed distributions for new comets should agree.

In Sect. 4.1, we found that there could have been $w_{j} N_{\mathrm{nf} ; j}$ new comets with given $\log (a)_{j}$, which are hypothetically observed in the $\mathrm{ZV}$ during the integration period. For every group with the given $\log (a)_{j}$, we can construct the distribution of each angular element. Summing then through the intervals of $\log (a)$ with the determined relative numbers of comets, i.e. those with $j=3,4,5,6$, and 7 , we can also construct the angular-element distributions for new comets in the assumed hypothetical set.

To estimate the size of the deficit of the relative numbers of comets in intervals $j=1$ and 2 , let us also sum the distributions of the angular elements through $j=1,2,3, \ldots, 7$. So, we obtain two series of results, the first series for a narrower $(\mathrm{N})$ interval of $\log (a)(4.55<\log (a)<5.05)$ and the second for a wider (W) interval of $\log (a)(4.35<\log (a)<5.05)$. Specifically, for both the intervals, we construct the theoretical distributions of $\omega, \Omega$, and $\cos (i)$ of new comets, as well as analogous distributions of new comets selected from every considered sample/subset of orbits known from observations.
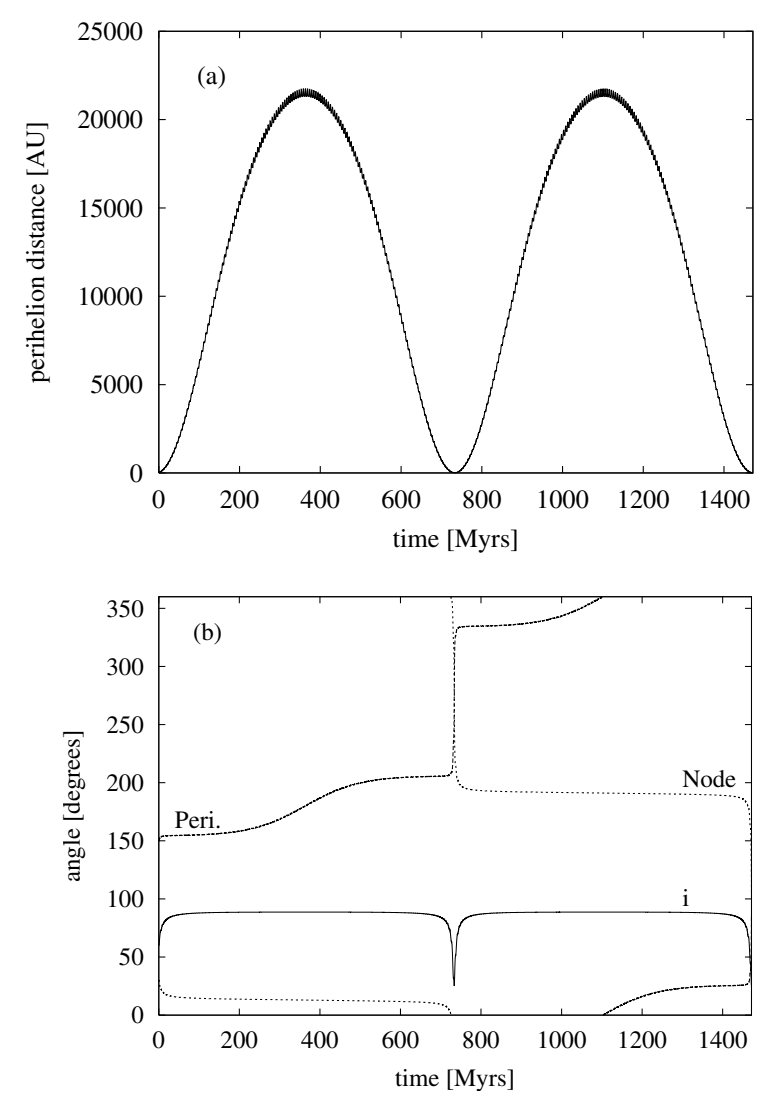

Fig. 4. The evolution of perihelion distance (a)) and galactic angular elements (b)) of a typical Oort-cloud comet during two libration cycles of its perihelion distance. Only the $z$-term of the Galactic-disc perturbative force was considered in the numerical integration of the orbit of this comet.

When constructing the distribution of an angular element, the following difficulty appears. In Fig. 4, the behaviour of four orbital elements, $q, \omega, \Omega$, and $i$, within two libration cycles of perihelion is demonstrated for a typical outer-OC comet. Such behaviour was revealed earlier by several authors (e.g. Heisler \& Tremaine 1986; Pretka \& Dybczyński 1994). It is clear that a new comet comes into the $\mathrm{ZV}$ just in that phase of the libration cycle (Fig. $4 \mathrm{a}$ ) when $q$ is at its minimum. In Fig. $4 \mathrm{~b}$, we can see that all angular elements change their values extremely in this phase. Therefore, the distributions of these elements for the current perihelion passage show rather chaotic behaviour. We propose that information on the actual distributions can be gained constructing the distributions for the previous perihelion passage.

A measure of agreement between the theoretical and observed distributions of given angular element is evaluated with the help of the root of mean squares $\chi$. For the $\mathrm{N}$-interval, this quantity varies in the intervals $(0.051,0.067),(0.040,0.060)$, and $(0.048,0.091)$ for $\omega, \Omega$, and $\cos (i)$ distributions, respectively. The respective intervals for the $\mathrm{W}$-interval of $\log (a)$ are $(0.066,0.069),(0.033,0.057)$, and $(0.023,0.063)$. From the found values, several partial conclusions can be drawn.

When the values of $\chi$ for $\omega, \Omega$, and $\cos (i)$ are added, the sums for the $\mathrm{W}$-interval are lower than that for the $\mathrm{N}$-interval. It indicates that the relative deficit of comets in the $j=1$ and $j=2$ intervals of $\log (a)$ is probably not very high. It biases the 
data to a lesser degree than the lower number of comets in the narrower interval.

Though the CCO sample cannot be statistically processed due to the absence of the determination error, the best agreement can be seen just for this most numerous data sample. Worse agreement between the theoretical and observed distributions can be observed using subset CL1 than using the entire sample (ALL).

The absolutely best agreement for the $\omega$ distribution is reached for the $\mathrm{N}$-interval using the CCO-ALL sample, and the absolutely best agreement for the $\Omega$ and $\cos (i)$ distributions in the $\mathrm{W}$-interval also uses the entire (ALL) CCO sample. A comparison of these theoretical and observed distributions is illustrated in Fig. 5, plots a, b, and c, respectively. The occurrence of the best agreement, when the CCO-ALL sample is used, proves that this sample is the most appropriate of the considered samples, despite the lack of statistical processing. It also documents a natural expectation that using a more numerous sample, one obtains a better agreement between observation and theory.

Let us discuss the main features of the obtained bestagreement distributions. In Fig. 5a, one can see that no observed new comet at its previous perihelion passage had a modified-galactic argument of perihelion in the intervals from about $60^{\circ}$ to $180^{\circ}$ and from about $270^{\circ}$ to $360^{\circ}$ (solid-line bars). This is quite good agreement with the theoretical prediction that says (dashed-line bars) new comets should not be observed at their previous perihelion passage in the intervals of $\omega$ from $90^{\circ}$ to $180^{\circ}$ and from $270^{\circ}$ to $360^{\circ}$. The least variable behaviour is predicted by theory for the modified-galactic longitude of ascending node plotted in Fig. 5b, and the prediction agrees relatively well with the observed distribution in this respect. From Fig. 5c, we can see that comets in orbits highly inclined to the Galactic equatorial plane at the previous perihelion passage dominate among the new comets. The peak at $\cos (i) \sim 0$ obviously appears due to conservation of the $z$-component of angular momentum (quantity $\sqrt{q}|\cos (i)|$ is approximately constant). It is known that the distribution of $\cos (i)$ of new comets at the current perihelion passage is roughly constant (e.g. Wiegert \& Tremaine 1999, Fig. 8b). A larger $q$ at the previous perihelion passage necessarily implies the decrease in $|\cos (i)|$.

\subsection{A lower estimate of the total population of the outer $O C$}

In Sect. 3.3, we documented a quasi constant distribution of perihelion distance of new comets in the $\mathrm{ZV}$ in the $\mathrm{N}$-interval of $\log (a)$. The population of the part of OC in this interval can be derived assuming a constant distribution of $q$ for all LP comets. Then, new comets with $a$ within the $\mathrm{N}$-interval and with $q$ within a given interval of heliocentric distance in the $\mathrm{ZV}$ represent a certain amount of all LP comets in the given interval of $q$, so we can expect that both new-comet-withinthe-N-interval and all-LP-comet samples are proportionally biased by the selection effects. Their ratio must be the same for each equidistant interval of $q$. A detailed discussion concerning the distribution of LP-comet $q$ in the $\mathrm{ZV}$ was given by
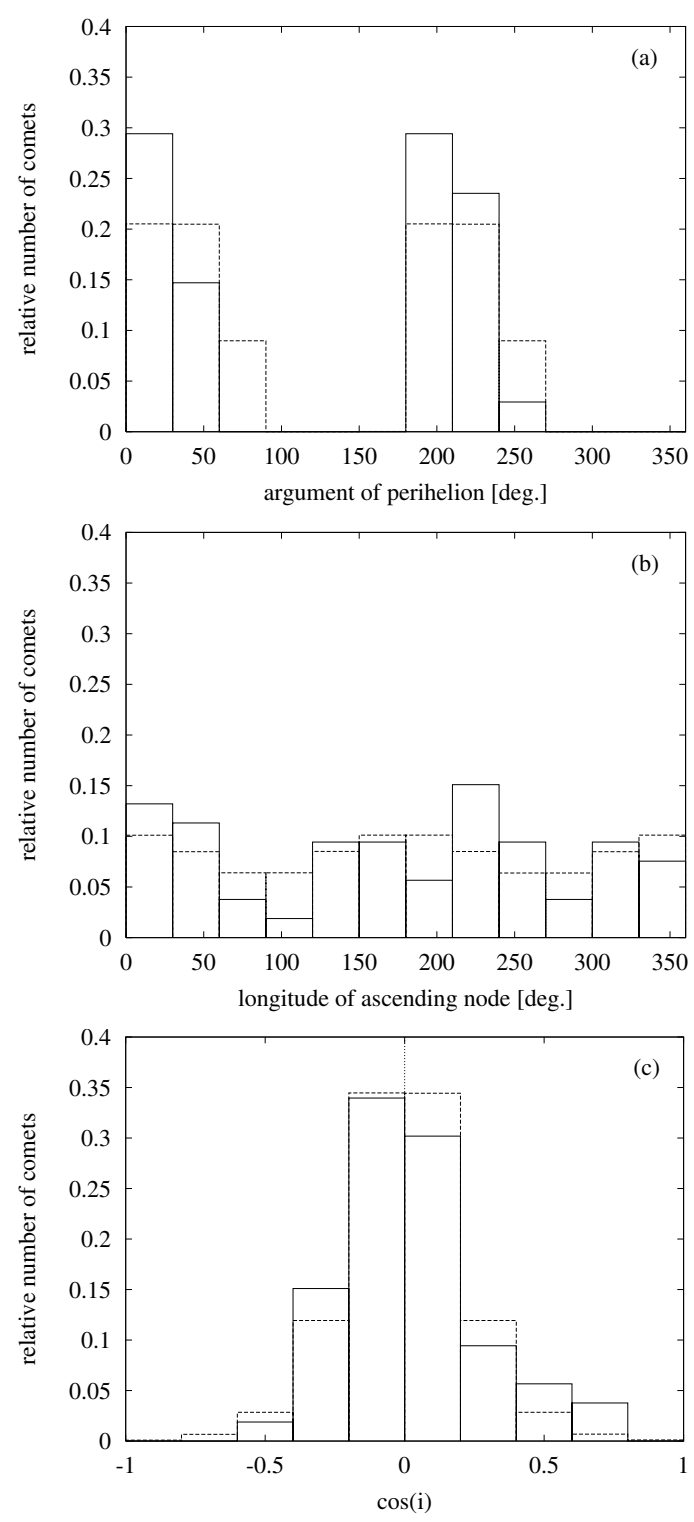

Fig. 5. The actually observed (solid-line bars) and theoretical (dashedline bars) relative distributions of $\omega(\mathbf{a})), \Omega(\mathbf{b}))$, and $\cos (i)$ (c)) for original orbits of new comets at their previous perihelion passage in the modified galactic coordinate system. The distributions were constructed for two, GAC and CCO, samples of observational data, the whole sets as well as the subsets - see Sect. 3.2. The above plots illustrate the best obtained agreement between observation and theory.

Wiegert \& Tremaine (1999) and can also be found in Paper I. Two extreme cases are constant and linear distributions. Assuming the constant distribution, we can give a lower estimate of LP comets in the $\mathrm{ZV}$ and, consequently, in the $\mathrm{N}$ interval of the OC.

A relation for calculation of the total population $N_{\text {tot }}$ can be derived in the same way as Eq. (5) for calculating the number of comets in the $j$ th interval of $\log (a)$. Omitting subscript $j$ in Eq. (5), we can write

$N_{\mathrm{tot}}=\frac{1}{\eta} \frac{t_{\mathrm{IP}}}{t_{\mathrm{OP}}} \frac{N_{\mathrm{all}}}{N_{\mathrm{nf}}} n_{\mathrm{obs}}$.

This time, $\eta$ is rate of completeness of comets from the $\mathrm{N}$-interval in the catalogue, $N_{\text {all }}$ the number of considered 
Table 4. The lower estimate for the total population of the outer OC $N_{\text {tot }}$ in its parts corresponding to the $\mathrm{N}$ and W intervals of $\log (a)$ (Sect. 4.2) as determined on the basis of several subsets of data (Sect. 3.2). $N_{\mathrm{nf}}$ is the number of predicted new comets in the $\mathrm{ZV}$ assuming the constant distribution of $\log (a)$ in the outer OC; $n_{\mathrm{obs}}$ is the number of new comets with known original orbit in the given subset of data; $n_{\text {theo }}$ is the theoretical number of all LP comets in the given subset, if it were complete; $n_{\text {act }}$ is the actual number of all LP comets in the subset as counted from the data, and $\eta$ rate of completeness of the given subset of the used data.

\begin{tabular}{lcccccc}
\hline \hline subset & $N_{\text {nf }}$ & $n_{\text {obs }}$ & $n_{\text {theo }}$ & $n_{\text {act }}$ & $\eta$ & $\begin{array}{c}N_{\text {tot }} \\
{\left[10^{11}\right]}\end{array}$ \\
\hline GAC & \multicolumn{7}{c}{ N-interval } \\
-ALL & 4079 & 16.94 & 2856 & 250 & 0.0875 & 1.0 \\
-CL1 & 4079 & 15.70 & 2856 & 140 & 0.0490 & 1.7 \\
CCO & & & & & & \\
-ALL & 5484 & 34 & 4000 & 374 & 0.0935 & 1.3 \\
-CL1 & 5484 & 31 & 4000 & 238 & 0.0595 & 1.9 \\
\hline GAC & \multicolumn{7}{c}{ W-interval } \\
-ALL & 7695 & 24.09 & 2856 & 250 & 0.0875 & $<1.1$ \\
-CL1 & 7695 & 22.22 & 2856 & 140 & 0.0490 & $<1.8$ \\
CCO & \multicolumn{7}{c}{} & & & & \\
-ALL & 10556 & 52 & 4000 & 374 & 0.0935 & $<1.5$ \\
-CL1 & 10556 & 48 & 4000 & 238 & 0.0595 & $<2.1$ \\
\hline
\end{tabular}

hypothetical comets in the N-interval, $N_{\mathrm{nf}}$ the number of predicted new comets in the $\mathrm{ZV}$ assuming a constant distribution of $\log (a)$ in the $\mathrm{N}$-interval, and $n_{\mathrm{obs}}$ the number of new comets with known original $\mathrm{N}$-interval orbit in the catalogue. Assuming proportionality between new comets from the Ninterval in the $\mathrm{ZV}$ and all LP comets in the $\mathrm{ZV}, \eta$ also gives the completeness of all LP comets with $q$ in the ZV in the catalogue. The $\mathrm{N}$-interval covers $j=3$ to 7 . Summing the appropriate $N_{\mathrm{all} ; j}, N_{\mathrm{nf} ; j}$ (for CCO-ALL), and $n_{\mathrm{obs} ; j}$ (for CCO-ALL) given in Tables 1 and 2 , we can easily find $N_{\text {all }}=72000$, as well as the values of $N_{\mathrm{nf}}$ and $n_{\mathrm{obs}}$, which are given in the first part of Table 4 . We saw $t_{\mathrm{OP}}=168$ and 181 years for the GAC and CCO sample, respectively. $t_{\mathrm{IP}}=5 \mathrm{Gyr}$.

The largest uncertainty when estimating the total population comes from poor knowledge of $\eta$. In the following, we attempt to estimate it for the assumed constant distribution of $q$ in the ZV. In the Catalogue of Cometary Orbits (Marsden $\&$ Williams 2003), there are 1516 individual orbits of singleapparition comets. A lot of them are fragments of split nuclei, therefore a homogenization of the set is necessary. After excluding the sungrazing comets (except for representatives of independent groups), fragments of observed splittings and comets suspected of being such fragments, one gains a homogenized set of 783 orbits of LP comets (Paper I, Sect. 2.1). Making the same considerations as in Paper I, Sect. 2.1, we can conclude that about $90 \%$ of all comets with perihelia within 1 AU have been discovered during the last 20 years.

The frequency of cometary perihelion passages at the constant $q$ distribution can be determined from the fact that 49 LP comets with $0.2<q<1.0 \mathrm{AU}$ have been observed at their return to the Sun within the last 20 years. In this context, we do not consider comets with $q<0.2 \mathrm{AU}$, because it is impossible to perfectly eliminate the fragments of split sungrazers with a very small $q$. Taking the $90 \%$ efficiency of discoveries into account, we can easily calculate the frequency of perihelion passages per year for comets with $0<q<1.0 \mathrm{AU}$ : $\approx 3.4$ year $^{-1} \mathrm{AU}^{-1}$.

For all considered sets and subsets of original orbits of LP comets, the values of $\eta$, as well as the resulting lower estimates of the total population, are given in Table 4. For example, the CCO-ALL sample of original cometary orbits spans a period $t_{\mathrm{OP}}=181$ years, and the adopted border of the $\mathrm{ZV}$ is $6.5 \mathrm{AU}$; therefore the sample in the catalogue should contain 4000 comets, if it were complete. Since it actually contains only $n_{\text {act }}=374$ comets with $q<6.5 \mathrm{AU}, \eta \approx 0.094$. For this value of the completeness rate, the total population $N_{\text {tot }}$ of the part of the outer OC in $4.55<\log (a)<5.05$ is larger than about $1.3 \times 10^{11}$. In general, $N_{\text {tot }}$ is roughly $1 \times 10^{11}$ to $2 \times 10^{11}$.

Several authors in the past have published estimates of the total population of the entire observable outer OC. To enable a consistent comparison of our result with these estimates, we repeat the estimate of $N_{\text {tot }}$ for the W-interval, too. The corresponding $N_{\text {all }}=100800$ and $N_{\text {nf }}, n_{\text {obs }}$ can be found in the second part of Table 4 . Since the contributions for $j=1$ and 2 are underestimated, the values of $N_{\text {tot }}$ given in the last column of the second part of Table 4 are even smaller for the W-interval than the actual lower estimates would be. An increase of $N_{\text {tot }}$ for the $\mathrm{W}$-interval with respect to the $\mathrm{N}$-interval is not very significant; therefore we again state that $N_{\text {tot }} \approx 1 \times 10^{11}$ to $2 \times 10^{11}$.

The lower limit found is consistent with the latest estimate of the total population of the outer OC done by Heisler (1990), who presented the value of $5 \times 10^{11}$ comets with $a>20000$ AU.

\subsection{Depletion of the outer Oort cloud by the Galactic tide}

The force from the GT with secondary terms results in only a minute change in the semi-major axis of an OC comet. This force alone cannot deplete the distant reservoir. It can, however, reduce the cometary perihelia and cause comets with perihelia otherwise detached from the planetary region to become objects of erosive planetary perturbation. In an attempt to estimate the erosion of the OC, we must bear in mind that the Solar System has moved from its beginning through a tinier and denser Galactic environment with a different value of mean mass density. Moreover, the assumed homogenity of this environment could have been broken several times by a near accumulation of matter in a Galactic arm. The perturbative force of the GT has been variable. We can also expect that other outer perturbers have probably become dominant many times, not only depleting but replenishing the outer cloud from the inner reservoir as well. Therefore, a variable rate of OC depletion can be expected over a long time scale.

Nevertheless, it can be useful to estimate the rate of depletion of the OC under the assumption of a single, constant GT perturbation. Though such information cannot be regarded as reliable on a very long time scale, it can perhaps clarify some aspects of the recent evolution of the outer OC. 


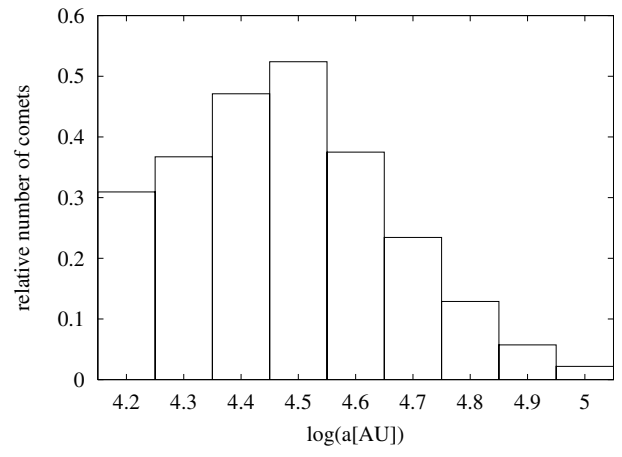

Fig. 6. The relative amount of comets lost from the Oort cloud (from its part with $a>14125 \mathrm{AU}$ ) due to combined Galactic-tide-planetary perturbation.

On the basis of the numerical integration performed, we can find the relative amount of comets with given $\log (a)$ that have entered the perturbative region of planets below $15 \mathrm{AU}$. Specifically, we count the numbers of $q$-libration cycles with minimum $q$ less than $15 \mathrm{AU}$. Some representative comets thus can be counted two or more times, at the repeating returns of their perihelia to the ZV during 5 Gyr. All such comets can in practice be regarded as lost. Weissman (1979) found that Jupiter and Saturn removed almost all the comets having perihelia in their region from the $\mathrm{OC}$ at the first perihelion passage.

The relative amounts of comets entering the planetary perturbative region for every considered value $\log (a)$ are demonstrated in Fig. 6. In total, about $19 \%$ comets would have been lost due to this depletion mechanism in the outer OC, $4.35<$ $\log (a)<5.05$, during the age of the Solar System, if the depletion rate were constant. The ratio of comets lost from the outer OC due to the Galactic-tide-planetary (GTP) perturbation and new comets that enter into the $\mathrm{ZV}$ in the same time frame is $\approx 2.5$.

Consulting the plots in Fig. 3, plots c, d, and Fig. 6, we can speculate that the behaviour of the number of comets, derived from the CCO sample (Fig. 3, plots c and d), qualitatively anticorrelates with the behaviour of the erosion of the outer OC (Fig. 6) in the interval $4.35<\log (a)<4.65$ : the minimum at the value $\log (a)=4.5$ in Fig. 3, plots $\mathrm{c}$ and d, corresponds to the maximum of this value in Fig. 6. The observed local minimum in Fig. 3, plots $\mathrm{c}$ and d, could not necessarily be the result of observational selection creating a lack of comets in the catalogue.

As mentioned above, the OC has not been depleted exclusively by GTP perturbation. Considering the result by Heisler \& Tremaine (1986), it does, however, seem that the mean effect of stellar perturbations has acted in a similar way and can be regarded as a simple magnification of the GTP perturbation. Heisler \& Tremaine, performing the Monte Carlo simulation, confirmed that the mean effect of stellar encounters on the orbital evolution in the $\mathrm{OC}$ is the same as that of a GT of the same density. Of course, an individual orbit does deviate substantially from the contour derived for the smooth tidal force.

The shortest semi-major axes of orbits we numerically integrated were $\log (a)=4.2$. The continuous decrease in the depletion rate for $\log (a)<4.5$ (Fig. 6) obviously goes on below $\log (a)=4.2$. The non-zero depletion rate in a region inside the inner boundary of the outer OC implies that the GT, in combination with Jupiter and Saturn, have depleted a significant part of the inner OC as well.

\section{Some additional discussion}

\subsection{On the existence of the inner Oort cloud (Hills cloud)}

The distribution of semi-major axis peaks at $\log (a) \approx 4.6$, when constructed using the GAC and CCO samples (Fig. 3, plots a-d). For a shorter $a$, as this quantity decreases, the number of comets decreases. Using the CCO sample (Fig. 3, plots c and d), another peak in the semi-major axis distribution occurs at $\log (a) \approx 4.4$. But for neither this sample nor the GAC sample have any new comets been observed in the $\mathrm{ZV}$ inside a border of about $\log (a)=4.35(\approx 22400 \mathrm{AU})$. With respect to accepted concepts of the Oort cloud origin, a real-cometarypopulation cut off at this value of $a$ would be unnatural. The absence of new comets with $a<20000 \mathrm{AU}$ in an observed sample was first noticed by Hills (1981), who claimed that it is not proof of the actual non-existence of such bodies in the OC. Using our data, we can actually confirm that this cut-off is only apparent. The GT is able to reduce $a$ of comets enough to overcome the Jupiter-Saturn barrier only for $a \geq 22400 \mathrm{AU}$ as documented in Table 2 by an abrupt decrease in the number of hypothetical new comets between $\log (a)=4.4(j=1)$ and $\log (a)=4.3(j=0)$ for the constant distribution of $\log (a)$. Specifically, this number decreases from 1072 to 0 for the bodies corresponding in the ZV to the GAC sample, and from 1696 to 8 for those corresponding in the $\mathrm{ZV}$ to the CCO sample.

The probable existence of the inner, Hills cloud (HC, hereinafter) is indicated in Fig. 3, plots c and d, by the considerable increase in the number of comets at the inner boundary of the outer cloud, $\log (a) \approx 4.4$. The peak is not seen in plots a and b of Fig. 3, where the distributions were constructed using the GAC sample containing a much lower number of comets with the largest known perihelion distances than the CCO sample. From the character of GT perturbation, it is clear that larger perihelion distances of new comets allow increased amounts of new comets with shorter and shorter semi-major axes in a given cometary sample. In other words, if we have the data on new comets with a larger $q$, we can study a nearer-to-Sun part of the OC. While no new comets with $q>5 \mathrm{AU}$ are contained in the GAC sample, new comets up to $q=6.5 \mathrm{AU}$ are in the $\mathrm{CCO}$ sample, so we have better quality information about the part of OC corresponding to $\log (a) \approx 4.4$ from the CCO sample.

\subsection{The upper acceptable limit of the total cometary population}

The acceptable scenarios of the OC origin are, at present, those assuming its formation within the formation of the Solar System itself. This fact implies a limitation on the total mass of the cometary cloud. Providing an estimate of the cloud population, it is necessary to check if the corresponding mass lies within the reasonable limit. In Paper I, we concluded that older 
estimates of the total mass of cometary population have resulted in too high and problematic a value. The problem is referred to as the high-mass problem. In this subsection, we find a minimum value for the total mass of the whole cometary population and discuss it with respect to the deduced acceptable upper limit.

This total mass of cometary population at present can simply be calculated as the product of the number of cometary nuclei in the OC and the typical mass of one cometary nucleus. The masses of a lot of individual cometary nuclei have been determined in recent decades. Summarizing these observational determinations, Neslušan (2003) gave the typical or average mass of an LP-comet nucleus of $1 \times 10^{16} \mathrm{~kg}$, assuming a nominal mean density of cometary nucleus $\rho_{5}=500 \mathrm{~kg} \mathrm{~m}^{-3}$. This nominal value of mean density is also assumed throughout this paper and for a mean density $\rho_{\mathrm{N}}$ other than the nominal mean density, it is necessary to correct all following minimum estimates of mass by a factor $\rho_{\mathrm{N}} / \rho_{5}$.

The value of the typical mass of an LP-comet nucleus can, however, be affected by a selection effect: we can expect that observers have chosen the brightest and, therefore, largest comets to measure their size. No such effect can be expected in the case of short-period comets with an average mass of $2 \times 10^{14} \mathrm{~kg}$, as the sizes of about $2 / 3$ short-period cometary nuclei are known and included in the Neslušan analysis. The rest of the short-period cometary nuclei are prevailingly those of the most recently discovered comets with large perihelion distances. Since it was revealed, regardless of observational selection, in the above work that LP-comet nuclei are, on average, larger than their short-period counterparts, the value of $2 \times 10^{14} \mathrm{~kg}$ can be regarded as a lower limit to the typical mass of LP-comet nucleus. We note the minimum mass of $2 \times 10^{14} \mathrm{~kg}$ is five times larger than the recently frequently used typical mass of cometary nucleus $4 \times 10^{13} \mathrm{~kg}$ derived by Weissman (1996). He related the absolute magnitude of a comet to cometary mass. Such a relation can be expected, however, to contain a larger degree of uncertainty than the values derived from more or less direct determinations of the size of cometary nuclei.

We would like to emphasize that the minimum value, used here, of typical mass of LP-comet nucleus is exclusively related to the bodies observed in the $\mathrm{ZV}$ and, thus, compatible with the estimated population of the OC, which is also gauged with the help of the same category of bodies. One cannot, of course, reject the possible existence of some smaller cometary nuclei that are too faint to be observable in the $\mathrm{ZV}$, which can decrease the average mass of the LP-comet nuclei. In such a case, the gauging must, however, result in a larger number of bodies in the distant reservoir. In the given context, we can ignore this partial problem because additional bodies could, in principle, only increase the total mass of the OC. As we are attempting to find a minimum total mass, it is not necessary to include a possible unknown mass increment.

The upper limit of acceptable total mass of the cometary population depends on a specific concept of comet origin. If we assume formation of comets in the proto-planetary disc is a byproduct of planet formation, then the following consideration is relevant.
The relative abundance of heavy chemical elements (all elements apart from hydrogen and helium) in the Sun is well known to be $2 \%$ of solar mass (e.g. Roxburgh 1985). There is no reason to assume a different chemical composition of the gaseous-dusty proto-solar nebula, from which the proto-sun and proto-planetary disc formed. Its heavy-chemical-element (HCE) fraction had to represent no more than $0.02 M_{\mathrm{N}}$, where $M_{\mathrm{N}}$ is the total mass of the nebula. Since $2.8 \times 10^{-4} M_{\odot}$ of HCE mass is locked in the planets, the total $0.02 M_{\mathrm{N}}-2.8 \times 10^{-4} M_{\odot}$ of HCE mass belonged to the disappeared dust component and all small bodies once created in the Solar System. A concept of low mass proto-solar nebula assumes $M_{\mathrm{N}} \approx 0.05 M_{\odot}$. For this value of $M_{\mathrm{N}}$, one can calculate $0.02 M_{\mathrm{N}}-2.8 \times 10^{-4} M_{\odot} \approx$ $0.00072 M_{\odot}$, i.e. about $240 M_{\oplus}$ or $3 / 4$ of the mass of Jupiter. If we omit the negligible total mass of the dust and main-belt asteroids, the latter can be regarded as the total HCE mass of the whole cometary population formed in the proto-planetary disc.

In a cometary nucleus, the helium component represents a negligible fraction of its total mass. The majority of hydrogen is locked in water ice, where it represents $11 \%$ of the mass. According to the generally accepted model of cometary nucleus known as "dirty snowball", we can expect that the dusty, rocky, and possibly metal admixture to the water ice increases the abundance of HCEs in the nucleus; therefore the fraction of hydrogen-helium component can represent $\approx 10 \%$ of nuclear mass in maximum. A minimum estimate of the HCEcomponent mass in comets can thus be estimated by multiplying their total mass by a factor of 0.9 .

Taking into account both our lower estimate of the total number of outer-OC comets of $1 \times 10^{11}$ to $2 \times 10^{11}$ and the minimum typical mass of LP-comet nucleus of $2 \times 10^{14} \mathrm{~kg}$, the minimum total mass of the outer-OC population is about $3.3 M_{\oplus}$ to $6.7 M_{\oplus}$ at present. Assuming a loss of $19 \%$ by the GTP perturbation as a typical loss over the age of the Solar System, the initial total mass of the outer OC had to be larger than about $4.1 M_{\oplus}$ to $8.2 M_{\oplus}$.

Considering the minimum total mass of the outer OC, let us estimate the mass of the whole OC a short time after its creation within the recent reference model of OC by Dones et al. (2004), at least. The authors show that $\approx 5 \%$ bodies reside in the OC today, whereby about a half $(\approx 2.5 \%)$ is situated in its outer part ("cold runs"). Taking these figures into account, our estimate of the total mass of the outer OC has to be enlarged by a factor of $36(0.9 / 0.025)$ to obtain the mass of the HCE component of the maximum cometary population that ever existed in the distant cloud. For a minimum typical mass of cometary nucleus of $2 \times 10^{14} \mathrm{~kg}$, the minimum total mass of the HCE component is about 120 to $240 M_{\oplus}$ for our minimum estimate of the present total mass of the outer OC. The value approaches the acceptable upper limit. The minimum value obtained for "warm runs", where about $\approx 1.7 \%$ of bodies is situated in the outer OC today, is $\approx 170$ to $\approx 350 M_{\oplus}$, also close to the upper limit.

The minimum values found for the total mass do not seem to be very problematic, when compared to the acceptable upper limit of $240 M_{\oplus}$. We must however remember that these values are related only to the distant cloud or its HCE component. According to proto-planetary-disc comet origin, this cloud was 
created by bodies once ejected from the region of giant planets. The process of ejection was relatively inefficient and the prevailing part of the ejected bodies escaped into an interstellar space (and a fraction was thrown into the Sun); so the cometary population originally created in the proto-planetary disc had to be several times larger, exceeding the acceptable upper limit of the HCE component in the disc.

Because of the high-mass problem, let us also consider an alternative scenario of comet origin when the prevailing part of the comet cloud is a relict of a proto-solar nebula from an era before its collapse (Neslušan 2000). The mutual adhesion of slow heavy-atom nuclei and molecules is much higher than that of hydrogen and helium nuclei in nebular regions, which are the birthplaces of new stars. The dust grains and larger bodies created in such an environment must preferably consist of HCEs. Consequently, a significant part of these elements can be locked in macroscopic, comet-nucleus-sized bodies. While the populations of objects in the proto-planetary disc had to be created with the same kind of material as the Sun and, consequently, had to initially contain the same $2 \%$ amount of HCEs, the natal cloud of the Solar System could have had an extra, macroscopic, HCE component. Therefore, the limitation on the total mass of OC valid for the proto-planetary-disc origin, is not valid for the alternative concept. Here, another limitation can be derived. It is known that interstellar matter consists not only of gas with $2 \%$ of HCEs, but of dust as well. The latter is created predominantly by HCEs and represents $\approx 1 \%$ of the matter (Holzer 1989). Unfortunately, there is no indication of what amount of the "dust" component could be concentrated in macroscopic cometary nuclei in the natal cloud of the Solar System. One can guess that the $\approx 1 \%$, i.e. $\approx 3300 M_{\oplus}$, is an upper limit.

Consulting plot $\mathrm{c}$ or $\mathrm{d}$ of Fig. 3 in this work and Figs. $2 \mathrm{a}-\mathrm{f}$ in Neslušan (2000), we see that the relict-origin models of the $\mathrm{OC}$ with an initial velocity distribution peak $v_{\mathrm{P}} \lesssim 0.7 v_{\mathrm{I}}$ disagree with observation and have to be discarded. There are too few comets with $\log (a) \geq 4$. 2 . Even the distributions of $\log (a)$ in the models with a higher $v_{\mathrm{P}}$ do not quantitatively agree with the actual distribution very well, but the behaviour depends on free input parameters, and an agreement can probably still be reached.

The models with $v_{\mathrm{P}}=0.75 v_{\mathrm{I}}$ contain about $10 \%$ of comets with $\log (a) \geq 4.35$ and less than half of the objects were incorporated into the proto-planetary disc. Again considering the erosion rate $19 \%$ as typical over the age of Solar System and minimum average mass of one cometary nucleus, $2 \times 10^{14} \mathrm{~kg}$, the minimum total mass of whole original population was about 82 to $160 M_{\oplus}$ for these models. This is a lower value than that derived from the proto-planetary-disc-origin concepts and much lower than the corresponding upper limit of $3300 M_{\oplus}$.

\subsection{The other perturbers of the Oort cloud}

Besides the GT, passing stars and interstellar molecular clouds are considered to significantly perturb the cometary orbits in the OC. According to Biermann \& Lüst (1978) and Biermann (1978) (see also Duncan et al. 1987), encounters with passing molecular clouds may have a perturbing influence on the comets that is comparable to that of stars. However, the influence of molecular clouds is difficult to estimate because their parameters are uncertain (Hut \& Tremaine 1985), and the consistency of observations with models of the comet cloud that neglect molecular clouds suggests that they do not have a major qualitative influence on the evolution of the comet cloud. The small influence of the interstellar clouds was also confirmed by Morris \& Muller (1986), who concluded that the frequency of comet showers induced by these clouds is much lower than that from passing stars. Since it appears that the tidal torque dominates stellar perturbations as a source of new comets, contributing roughly $80 \%$ of the flux of new comets (Duncan et al. 1987), the omission of molecular clouds in our study should not have a serious effect on the results.

The stellar perturbations on the OC were recently studied by Dybczyński (2002), who obtained several important results. A passing star compels a cometary shower in the ZV with a strongly asymmetric distribution of perihelia in the celestial sphere. During each stellar passage about 100 times more comets are lost than enter the $\mathrm{ZV}$, which means this ratio is 40 times higher than that in the case of combined GTP perturbation found in our work. In the case of the passage of a star with mass equal to $1 M_{\odot}$ and with relative velocity $20 \mathrm{~km} \mathrm{~s}^{-1}$, and a minimum Sun-star distance of $60000 \mathrm{AU}$, which can be regarded as a typical stellar passage, only 0.3 comets come to the $\mathrm{ZV}$ per year during the maximum of the appropriate shower. We note that Dybczyński defined the $\mathrm{ZV}$ as a region within 5 AU from the Sun. The total number of comets in the OC was scaled to be $10^{12}$. In a relatively close passage, when the minimum Sun-star distance is $30000 \mathrm{AU}$, the influx of comets in the $\mathrm{ZV}$ is 7 per year during the shower maximum.

It is clear that we do not observe any asymmetry in the directional distribution of perihelia of new comets in the present, which could be assigned to a cometary shower forced by a passing star in the not too distant past. Therefore, we are not "in shower" and, consequently, the rate of new comets sent to the $\mathrm{ZV}$ by passing stars can be assumed to be negligible in comparison with the rate of new comets sent to the ZV by the GT. Specifically, the first rate is at least one order lower than 0.3 comets per year. We prefer to consider the value 0.3 in opposition to 7, because a more distant stellar passage is more probable. The second rate is 17 comets per year within $5 \mathrm{AU}$, when the constant distribution of cometary $q$ is taken into account. It is reasonable to omit stellar perturbations when a sample of observed new comets in the $\mathrm{ZV}$ is analysed.

Obviously stellar perturbations cannot be neglected when estimating of the depletion of the $\mathrm{OC}$, as the ratio of lost comets to those becoming visible in the $\mathrm{ZV}$ is 40 times higher for stellar perturbations in comparison with the GTP perturbation and the stellar depletion rate can rise considerably during very close stellar passages. It is, however, possible that stellar perturbations have caused a replenishment of the OC comparable to that by the GTP perturbation or even smaller over the age of the Solar System. In such a case, the reasoning in some earlier papers that a highly populated inner reservoir is necessary to replenish the outer OC would be irrelevant. According to the recent study by Dones et al. (2004), the total depletion rate 
is also lower than estimated earlier, about $28 \%$ (the decrease from $7.6 \%$ at $840 \mathrm{Myr}$ to $5.5 \%$ at $4 \mathrm{Gyr}$ ).

\section{Summary of the main conclusions on the outer Oort cloud}

Using the sample of 249 original orbits of LP comets with a known determination error of $1 / a$ enabling a statistical processing and 383 original orbits without any determination error (a statistically unprocessed sample), the following partial conclusions can be stated.

(1) For the value of mean mass density of the local Galactic environment $\rho=0.15 M_{\odot} \mathrm{pc}^{-3}$ the semi-major axes of dynamically new comets coming to the $\mathrm{ZV}$ are: $4.35<$ $\log (a)<5.05$. The amount of comets with $\log (a)>5.05$ is obviously small, but uncertain. Thus as the dynamically active, outer $\mathrm{OC}$ we regard just that part of the $\mathrm{OC}$ with the cometary semi-major axes within the specified interval.

(2) Dynamically new comets can be chosen reliably from a catalogue by their perihelion distance. The perihelion distance of the original orbit at the present perihelion passage should be shorter than a certain outer border (currently $6.5 \mathrm{AU}$ ) to eliminate observation selection effects as much as possible, and $q$ at the previous perihelion passage has to be larger than $15 \mathrm{AU}$.

(3) The distribution of semi-major axis was found by assuming an initially constant $\log (a)$ distribution and, then, identifying the theoretically predicted $\log (a)$ distribution of new comets with their observed $\log (a)$ distribution. From the currently available data on the original cometary orbits, the distribution of semi-major axis of the OC can only be sketched for the interval $4.55 \lesssim \log (a) \lesssim 5.05$. The distribution of perihelion distance of corresponding comets in the $\mathrm{ZV}$ is approximately constant and, thus, equally biased by the observational selection throughout the interval. The observed rate of new comets with smaller $a$ is underestimated in comparison to those with $a$ in the above specified interval.

(4) In the interval of $4.55 \lesssim \log (a) \lesssim 5.05$, the relative distribution of comets can be approximated by a power-law function $\mathrm{d} N \propto a^{-s} \mathrm{~d} a$, where $s \approx 0.62$ to 0.65 for the entire samples of available data, as well as for less numerous, statistically smoothed class-1-quality orbits, and $s \approx 0.80$ for the statistically non-processed, class- 1 orbits.

(5) The distributions of the angular elements of new comets at their previous perihelion passage can be used to evaluate the correctness of the outer-OC model (adopted initial conditions). The theoretical distributions can be constructed independently of their observed counterparts in this case. The best agreement between the observed and theoretical distributions of $\omega, \Omega$, and $\cos (i)$ is obtained for the CCO-ALL/N, CCO-ALL/W, and CCO-ALL/W sample/interval of $\log (a)$, respectively, where the N-interval is a narrower interval of $\log (a)(4.55<\log (a)<5.05)$ and the $\mathrm{W}$-interval is a wider interval of this quantity $(4.35<\log (a)<5.05)$. This supports the suggestion that new comets with $4.35<\log (a)<4.55$ are not all that underestimated in the CCO sample. At the same time, it documents how dependent the agreement between the observation and theory is on the completeness of the observational data. The root of mean squares of the best agreement is $0.051,0.033$, and 0.023 for the $\omega, \Omega$, and $\cos (i)$ distributions, respectively, thus implying $5.1 \%, 3.3 \%$, and $2.3 \%$ of unexplained residua. The largest value, $\approx 5 \%$, can be regarded as a characteristic of the degree of uncertainty for the outer-OC model.

(6) After an appropriate perturbation by the GT, comets approach the ZV upon orbits that are (i) preferably highly inclined to the Galactic equatorial plane and (ii) have an argument of perihelion in the interval from $0^{\circ}$ to $90^{\circ}$ or from $180^{\circ}$ to $270^{\circ}$ in the modified galactic coordinates.

(7) The lowest estimate of the population for the outer OC based on the constant distribution of $q$ of LP comets in the $\mathrm{ZV}$ is about $1 \times 10^{11}$ to $2 \times 10^{11}$. Because of a relatively large uncertainty, no difference in this estimate can be observed for different samples of gauging data. We find it impossible to give a more exact estimate of the population, even its lower limit, from the data currently available.

(8) The minimum total mass of the outer OC, corresponding to the minimum population estimate given in conclusion (7) and to the minimum typical mass of LPcomet nucleus of $2 \times 10^{14} \mathrm{~kg}$, implies the necessity of at least a few times larger amount of heavy chemical elements in the $0.05 M_{\odot}$-proto-solar nebula than $2 \%$. This causes a problem for the concept of proto-planetary-disc origin of comets.

(9) The completeness of the entire GAC LP-comet data (including LP comets through 1989) throughout the ZV up to $5 \mathrm{AU}$ is estimated to be about $9 \%$, when a constant distribution of $q$ of LP comets is assumed. It has remained practically the same during another 13 years of further observations in the entire CCO LP-comet catalogue with the enlarged $\mathrm{ZV}$ up to $6.5 \mathrm{AU}$. Since the constant $q$ distribution is a lower limitation of the real $q$ distribution, the actual completeness is probably lower.

(10) Besides other perturbers, the OC has been depleted by the combined GTP perturbation. We found that $19 \%$ of the total population would have been lost due to the GTP perturbation over the age of the Solar System, if the depletion rate were constant and equal to the current rate.

(11) The number of comets in the interval $4.45<\log (a)<$ 4.55 is lower than that in the interval $4.55<\log (a)<$ 4.65 , regardless of the chosen sample of gauging data. The rate of comets removed from the OC by the GTP perturbation peaks just at $\log (a) \approx 4.6$. Because of this circumstance, we cannot decide whether the above decrease is a consequence of observational selection effects, reducing the number of comets with shorter $a$ more, or if it is a real decrease that has occurred due to depletion caused by the GTP perturbation.

(12) The ratio of those comets lost from the outer OC due to the GTP perturbation and the new comets coming in the $\mathrm{ZV}$ during the same time period is 2.5 . 
(13) The influx found for new comets to the ZV due to the GT is one to three orders higher than the background (out of cometary "shower") flux due to stellar passages. The background flux is estimated from the fluxes at shower maximum obtained by Dybczyński (2002).

A large uncertainty in our results comes from the poorly known mean density of Galactic matter in the solar neighbourhood. Another study is needed to refine the results with respect to this uncertainty. The estimated total mass of the outer OC is also uncertain due to not knowing the typical mass of cometary nucleus very well and, mainly, poor knowledge of the completeness measure of the catalogue used with the original orbital data. Despite these uncertainties, the results presented refine our knowledge of the structure of the outer OC and give another constraint on models for cometary reservoir formation.

Acknowledgements. We thank Dr. Piotr A. Dybczyński for the stimulating discussion of several questions during the preparation of our work, as well as for providing us with the complete set of the original orbital elements of long-period comets. This work was supported by VEGA - the Slovak Grant Agency for Science, grant No. 4012.

\section{References}

Antonov, V. A., \& Latyshev, I. N. 1972, in The Motion, Evolution of Orbits, and Origin of Comets, ed. G. A. Chebotarev, E. I. Kazimirchak-Polonskaya, \& B. G. Marsden (Dordrecht: Reidel), 341

Bahcall, J. N. 1984, ApJ, 276, 169

Bailey, M. E. 1986, Nature, 324, 350

Biermann, L. 1978, in Astronomical Papers Dedicated to Bengt Strömgren, ed. A. Reiz, \& T. Anderson (Copenhagen: Copenhagen Obs.), 327

Biermann, L., \& Lüst, R. 1978, Sits. ber. Bayer. Akad. Wiss. Mat.Naturw., K1

Clube, S. V. M., \& Napier, W. M. 1982, QJRAS, 23, 45

Crézé, M., Chereul, E., Bienaymé, O., \& Pichon, C. 1998, A\&A, 329, 920

Dones, L., Levison, H., Duncan, M., \& Weissman, P. 2004, Icarus, submitted

Duncan, M., Quinn, T., \& Tremaine, S. 1987, AJ, 94, 1330

Dybczyński, P. A. 2001, A\&A, 375, 643

Dybczyński, P. A. 2002, A\&A, 396, 283

Dybczyński, P. A., \& Pretka, H. 1996, Earth Moon Planets, 72, 13

Everhart, E., \& Marsden, B. G. 1983, AJ, 88, 135
Everhart, E., \& Marsden, B. G. 1987, AJ, 93, 753

Fernández, J. A. 1994, in Asteroids, Comets, Meteors 1993, ed. A. Milani, M. Di Martino, \& A. Cellino (Dordrecht: Kluwer), Proc. IAU Symp., 160, 223

Fernández, J. A. 1997, Icarus 129, 106

Fernández, J. A. 2002, in Proc. Asteroids, Comets, Meteors, ACM 2002, ed. B. Varmbein (Noordwijk: ESA Publ. Div., ESTEC), ESA SP-500, 303

García-Sánchez, J., Weissman, P. R., Preston, R. A., et al. 2001, A\&A, 379,634

Harrington, R.S. 1985, Icarus, 61, 60

Heisler, J. 1990, Icarus, 88, 104

Heisler, J., \& Tremaine, S. 1986, Icarus, 65, 13

Heisler, J., Tremaine, S., \& Alcock, C. 1987, Icarus, 70, 269

Hills, J. G. 1981, AJ, 86, 1730

Holmberg, J., \& Flynn, C. 2000, MNRAS, 313, 209

Holzer, T. E. 1989, ARA\&A, 27, 199

Hut, P., \& Tremaine, S. 1985, AJ, 90, 1548

Jeans, J. H. 1919, MNRAS, 79, 408

Kerr, F.J., \& Lynden-Bell, D. 1986, MNRAS, 221, 1023

Marsden, B. G. 1990, AJ, 99, 1971

Marsden, B. G., \& Sekanina, Z. 1973, AJ, 78, 1118

Marsden, B. G., \& Williams, G. V. 2003, Catalogue of Cometary Orbits 2003, 15th edition (Cambridge: Smithson. Astrophys. Obs.)

Marsden, B. G., Sekanina, Z., \& Everhart, E. 1978, AJ, 83, 64

Matese, J. J., \& Lissauer, J. J. 2002, Icarus, 157, 228

Matese, J. J., Whitman, P. G., Innanen, K. A., \& Valtonen, M. J. 1995, Icarus, 116, 255

Morris, D. E., \& Muller, R. A. 1986, Icarus, 65, 1

Napier, W. M., \& Staniucha, M. 1982, MNRAS, 198, 723

Neslušan, L. 2000, A\&A, 361, 369

Neslušan, L. 2003, Contrib. Astron. Obs. Skalnaté Pleso, 33, 5

Neslušan, L., \& Jakubík, M. 2004, Contrib. Astron. Obs. Skalnaté Pleso, 34, 87 (Paper I)

Oort, J. H. 1950, Bull. Astron. Inst. Netherlands, 11, 91

Pretka, H., \& Dybczyński, P. 1994, in Dynamics and Astrometry of Natural and Artificial Celestial Bodies, ed. K. Kurzyńska, et al. (Poznań: Astron. Obs. A. Mickiewicz Univ.), 299

Roxburgh, I. W. 1985, Sol. Phys., 100, 21

Van Biesbroeck, G., Vesely, C. D., \& Marsden, B. G. 1974, AJ, 79, 1455

Weissman, P. R. 1979, in Dynamics of the Solar System, ed. R. L. Duncombe (Dordrecht: Kluwer), 277

Weissman, P. R. 1996, in Completing the Inventory of the Solar System, ed. T. W. Retting, \& M. Hahn (ASP), ASP Conf. Ser., 107, 265

Whitmire, D. P., \& Matese, J. J. 2003, Icarus, 165, 219

Wiegert, P., \& Tremaine, S. 1999, Icarus, 137, 84 